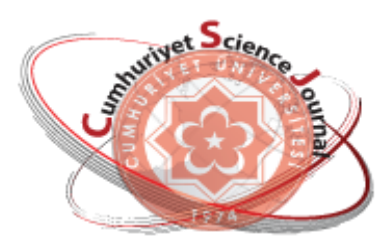

e-ISSN: 2587-246X

ISSN: $2587-2680$

\title{
A Theorem on Absolute Summability of Infinite Series
}

\author{
Bağdagül KARTAL
}

Erciyes University, Faculty of Science, Department of Mathematics, 38039, Kayseri, TURKEY

\begin{abstract}
In this paper, a theorem on absolute summability of infinite series is obtained by taking almost increasing sequence instead of positive non-decreasing sequence. Also, some results of absolute summability are given.
\end{abstract}

Keywords: Riesz mean, absolute summability, almost increasing sequence, Hölder inequality, Minkowski inequality.

\section{Sonsuz Serilerin Mutlak Toplanabilmesi Üzerine Bir Teorem}

Özet. Bu makalede, pozitif azalmayan dizi yerine hemen hemen artan dizi alınarak, sonsuz serilerin mutlak toplanabilmesi üzerine bir teorem elde edildi. Ayrıca, mutlak toplanabilme ile ilgili bazı sonuçlar verildi.

Anahtar Kelimeler: Riesz ortalaması, mutlak toplanabilme, hemen hemen artan dizi, Hölder eşitsizliği, Minkowski eşitsizliği.

\section{INTRODUCTION}

A positive sequence $\left(b_{n}\right)$ is said to be almost increasing if there exist a positive increasing sequence $\left(c_{n}\right)$ and two positive constants $K$ and $L$ such that $K c_{n} \leq b_{n} \leq L c_{n}$ [1]. Obviously, every increasing sequence is almost increasing. However, the converse need not be true as can be seen by taking the example, say $b_{n}=n e^{(-1)^{n}}$. Let $\sum a_{n}$ be an infinite series with its partial sums $\left(s_{n}\right)$. Let $\left(\varphi_{n}\right)$ be a sequence of positive real numbers. The series $\sum a_{n}$ is said to be summable $\varphi-\left|\bar{N}, p_{n} ; \delta\right|_{k}, \quad k \geq 1$ and $\delta \geq 0$, if [2]

$$
\sum_{n=1}^{\infty} \varphi_{n}^{\delta k+k-1}\left|\theta_{n}-\theta_{n-1}\right|^{k}<\infty
$$

where $\left(p_{n}\right)$ is a sequence of positive numbers such that

$$
P_{n}=\sum_{v=0}^{n} p_{v} \rightarrow \infty \quad \text { as } \quad n \rightarrow \infty \quad\left(P_{-i}=p_{-i}=0, i \geq 1\right)
$$

and 


$$
\theta_{n}=\frac{1}{P_{n}} \sum_{v=0}^{n} p_{v} s_{v}
$$

defines the sequence $\left(\theta_{n}\right)$ of the $\left(\bar{N}, p_{n}\right)$ mean of the sequence $\left(s_{n}\right)$ generated by the sequence of coefficients $\left(p_{n}\right)[3]$.

In the special case, if we take $\varphi_{n}=P_{n} / p_{n}$, then $\varphi-\left|\bar{N}, p_{n} ; \delta\right|_{k}$ summability reduces to $\left|\bar{N}, p_{n} ; \delta\right|_{k}$ summability [4]. Also, if we take $\varphi_{n}=P_{n} / p_{n}$ and $\delta=0, \varphi-\left|\bar{N}, p_{n} ; \delta\right|_{k}$ summability reduces to $\left|\bar{N}, p_{n}\right|_{k}$ summability [5]. Finally, if we take $\varphi_{n}=n, \delta=0$ and $p_{n}=1$ for all values of $n$, then we get $|C, 1|_{k}$ summability [6].

\section{KNOWN RESULTS}

Absolute summability methods are generally used to summability of an infinite series. There is an important application area of these methods. Especially, they have applications on different sequences such as positive non-decreasing, almost increasing and quasi power increasing sequences.

There are many different studies on absolute summability methods (see [2, 7-21]). Among them, in [7], the following theorem was proved.

Theorem 1 Let $\left(X_{n}\right)$ be a positive non-decreasing sequence and $\left(\beta_{n}\right),\left(\lambda_{n}\right)$ be sequences such that

$$
\begin{gathered}
\left|\Delta \lambda_{n}\right| \leq \beta_{n}, \\
\beta_{n} \rightarrow 0 \text { as } n \rightarrow \infty, \\
\sum_{n=1}^{\infty} n\left|\Delta \beta_{n}\right| X_{n}<\infty, \\
\left|\lambda_{n}\right| X_{n}=O(1) \text { as } n \rightarrow \infty
\end{gathered}
$$

hold where $\Delta \lambda_{n}=\lambda_{n}-\lambda_{n+1}$. If $\left(p_{n}\right)$ is a sequence of positive numbers such that

$$
\begin{gathered}
P_{n}=O\left(n p_{n}\right) \text { as } n \rightarrow \infty, \\
\sum_{n=1}^{m} \frac{p_{n}}{P_{n}}\left|s_{n}\right|^{k}=O\left(X_{m}\right) \text { as } m \rightarrow \infty,
\end{gathered}
$$

then the series $\sum a_{n} \lambda_{n}$ is summable $\left|\bar{N}, p_{n}\right|_{k}, k \geq 1$. 


\section{MAIN RESULT}

Theorem 1 is generalized as in the following form under weaker conditions by using an almost increasing sequence instead of a positive non-decreasing sequence.

Theorem 2 Let $\left(X_{n}\right)$ be an almost increasing sequence and $\varphi_{n} p_{n}=O\left(P_{n}\right)$. If conditions (4)-(8) of Theorem 1 and

$$
\begin{aligned}
& \sum_{n=1}^{m} \varphi_{n}^{\delta k-1}\left|s_{n}\right|^{k}=O\left(X_{m}\right) \text { as } m \rightarrow \infty, \\
& \sum_{n=v+1}^{m+1} \varphi_{n}^{\delta k-1} \frac{1}{P_{n-1}}=O\left(\varphi_{v}^{\delta k} \frac{1}{P_{v}}\right) \text { as } m \rightarrow \infty
\end{aligned}
$$

are satisfied, then the series $\sum a_{n} \lambda_{n}$ is summable $\varphi-\left|\bar{N}, p_{n} ; \delta\right|_{k}, \quad k \geq 1$ and $0 \leq \delta<1 / k$

Lemma 3 [22]. Under the conditions of Theorem 2, we have

$$
\begin{gathered}
n X_{n} \beta_{n}=O(1) \quad \text { as } n \rightarrow \infty, \\
\sum_{n=1}^{\infty} \beta_{n} X_{n}<\infty
\end{gathered}
$$

\section{PROOF OF THEOREM 2}

Let $\left(M_{n}\right)$ be the sequence of $\left(\bar{N}, p_{n}\right)$ mean of the series $\sum a_{n} \lambda_{n}$. Then, we get

$$
M_{n}=\frac{1}{P_{n}} \sum_{v=0}^{n} p_{v} \sum_{r=0}^{v} a_{r} \lambda_{r}=\frac{1}{P_{n}} \sum_{v=0}^{n}\left(P_{n}-P_{v-1}\right) a_{v} \lambda_{v}
$$

Now, for $n \geq 1$

$$
M_{n}-M_{n-1}=\frac{p_{n}}{P_{n} P_{n-1}} \sum_{v=1}^{n} P_{v-1} \lambda_{v} a_{v}
$$

From Abel's transformation, we obtain

$$
\begin{aligned}
M_{n}-M_{n-1} & =\frac{p_{n}}{P_{n} P_{n-1}} \sum_{v=1}^{n-1} P_{v} \Delta \lambda_{v} s_{v}-\frac{p_{n}}{P_{n} P_{n-1}} \sum_{v=1}^{n-1} p_{v} \lambda_{v} s_{v}+\frac{p_{n} s_{n} \lambda_{n}}{P_{n}} \\
& =M_{n, 1}+M_{n, 2}+M_{n, 3}
\end{aligned}
$$

To prove that $\sum a_{n} \lambda_{n}$ is summable $\varphi-\left|\bar{N}, p_{n} ; \delta\right|_{k}$, it is sufficient to show that

$$
\sum_{n=1}^{\infty} \varphi_{n}^{\delta k+k-1}\left|M_{n, r}\right|^{k}<\infty \text { for } r=1,2,3
$$


First, using the fact that $\varphi_{n} p_{n}=O\left(P_{n}\right)$ and the condition (4), we get

$$
\sum_{n=2}^{m+1} \varphi_{n}^{\delta k+k-1}\left|M_{n, 1}\right|^{k}=O(1) \sum_{n=2}^{m+1} \varphi_{n}^{\delta k-1} \frac{1}{P_{n-1}^{k}}\left\{\sum_{v=1}^{n-1} P_{v} \beta_{v}\left|s_{v}\right|\right\}^{k} .
$$

Now, using Hölder's inequality and the condition (8),

$$
\sum_{n=2}^{m+1} \varphi_{n}^{\delta k+k-1}\left|M_{n, 1}\right|^{k}=O(1) \sum_{n=2}^{m+1} \varphi_{n}^{\delta k-1} \frac{1}{P_{n-1}}\left\{\sum_{v=1}^{n-1} p_{v}\left(v \beta_{v}\right)^{k}\left|s_{v}\right|^{k}\right\}\left\{\frac{1}{P_{n-1}} \sum_{v=1}^{n-1} p_{v}\right\}^{k-1}
$$

Then, we obtain

$$
\sum_{n=2}^{m+1} \varphi_{n}^{\delta k+k-1}\left|M_{n, 1}\right|^{k}=O(1) \sum_{v=1}^{m} p_{v}\left(v \beta_{v}\right)^{k}\left|s_{v}\right|^{k} \sum_{n=v+1}^{m+1} \varphi_{n}^{\delta k-1} \frac{1}{P_{n-1}}
$$

Here, using the condition (11), we get

$$
\begin{aligned}
\sum_{n=2}^{m+1} \varphi_{n}^{\delta k+k-1}\left|M_{n, 1}\right|^{k} & =O(1) \sum_{v=1}^{m} \varphi_{v}^{\delta k} \frac{p_{v}}{P_{v}}\left(v \beta_{v}\right)^{k}\left|s_{v}\right|^{k} \\
& =O(1) \sum_{v=1}^{m} \varphi_{v}^{\delta k-1}\left(\frac{\varphi_{v} p_{v}}{P_{v}}\right)\left(v \beta_{v}\right)^{k}\left|s_{v}\right|^{k},
\end{aligned}
$$

then using the fact that $\varphi_{v} p_{v}=O\left(P_{v}\right)$, we obtain

$$
\sum_{n=2}^{m+1} \varphi_{n}^{\delta k+k-1}\left|M_{n, 1}\right|^{k}=O(1) \sum_{v=1}^{m} \varphi_{v}^{\delta k-1}\left(v \beta_{v}\right)\left(v \beta_{v}\right)^{k-1}\left|s_{v}\right|^{k}
$$

Now using the fact that the sequence $\left(X_{n}\right)$ is almost increasing and the condition (12), we obtain $\left(v \beta_{v}\right)^{k-1}=O(1)$. Thus, we have

$$
\sum_{n=2}^{m+1} \varphi_{n}^{\delta k+k-1}\left|M_{n, 1}\right|^{k}=O(1) \sum_{v=1}^{m} \varphi_{v}^{\delta k-1} v \beta_{v}\left|s_{v}\right|^{k}
$$

Then, using Abel's transformation, we get

$$
\sum_{n=2}^{m+1} \varphi_{n}^{\delta k+k-1}\left|M_{n, 1}\right|^{k}=O(1) \sum_{v=1}^{m-1} \Delta\left(v \beta_{v}\right) \sum_{i=1}^{v} \varphi_{i}^{\delta k-1}\left|s_{i}\right|^{k}+O(1) m \beta_{m} \sum_{v=1}^{m} \varphi_{v}^{\delta k-1}\left|s_{v}\right|^{k}
$$

Here considering the fact that

$$
\Delta\left(v \beta_{v}\right)=v \beta_{v}-(v+1) \beta_{v+1}=v \Delta \beta_{v}-\beta_{v+1},
$$

and using the condition (10), we have 


$$
\begin{aligned}
\sum_{n=2}^{m+1} \varphi_{n}^{\delta k+k-1}\left|M_{n, 1}\right|^{k} & =O(1) \sum_{v=1}^{m-1} v\left|\Delta \beta_{v}\right| X_{v}+O(1) \sum_{v=1}^{m-1} \beta_{v+1} X_{v+1}+O(1) m \beta_{m} X_{m} \\
& =O(1) \text { as } m \rightarrow \infty
\end{aligned}
$$

by (6), (13) and (12), respectively.

Now, again using the fact that $\varphi_{n} p_{n}=O\left(P_{n}\right)$ and Hölder's inequality, we have

$$
\begin{aligned}
\sum_{n=2}^{m+1} \varphi_{n}^{\delta k+k-1}\left|M_{n, 2}\right|^{k} & =O(1) \sum_{n=2}^{m+1} \varphi_{n}^{\delta k-1} \frac{1}{P_{n-1}^{k}}\left\{\sum_{v=1}^{n-1} p_{v}\left|\lambda_{v}\right|\left|s_{v}\right|\right\}^{k} \\
& =O(1) \sum_{n=2}^{m+1} \varphi_{n}^{\delta k-1} \frac{1}{P_{n-1}}\left\{\sum_{v=1}^{n-1} p_{v}\left|\lambda_{v}\right|^{k}\left|s_{v}\right|^{k}\right\}\left\{\frac{1}{P_{n-1}} \sum_{v=1}^{n-1} p_{v}\right\}^{k-1} .
\end{aligned}
$$

Then

$$
\sum_{n=2}^{m+1} \varphi_{n}^{\delta k+k-1}\left|M_{n, 2}\right|^{k}=O(1) \sum_{v=1}^{m} p_{v}\left|\lambda_{v}\right|\left|\lambda_{v}\right|^{k-1}\left|s_{v}\right|^{k} \sum_{n=v+1}^{m+1} \varphi_{n}^{\delta k-1} \frac{1}{P_{n-1}}
$$

Here, using the fact that the sequence $\left(X_{n}\right)$ is almost increasing and considering the condition (7), it is clear that $\left|\lambda_{v}\right|^{k-1}=O(1)$. Additionally, using the condition (11), we have

$$
\sum_{n=2}^{m+1} \varphi_{n}^{\delta k+k-1}\left|M_{n, 2}\right|^{k}=O(1) \sum_{v=1}^{m} \varphi_{v}^{\delta k-1}\left|\lambda_{v}\right|\left|s_{v}\right|^{k}
$$

Hence, we get

$$
\begin{aligned}
\sum_{n=2}^{m+1} \varphi_{n}^{\delta k+k-1}\left|M_{n, 2}\right|^{k} & =O(1) \sum_{v=1}^{m-1} \Delta\left|\lambda_{v}\right| \sum_{i=1}^{v} \varphi_{i}^{\delta k-1}\left|s_{i}\right|^{k}+O(1)\left|\lambda_{m}\right| \sum_{v=1}^{m} \varphi_{v}^{\delta k-1}\left|s_{v}\right|^{k} \\
& =O(1) \sum_{v=1}^{m-1} \beta_{v} X_{v}+O(1)\left|\lambda_{m}\right| X_{m} \\
& =O(1) \text { as } m \rightarrow \infty,
\end{aligned}
$$

by virtue of Abel's transformation, (4), (10), (13) and (7).

Finally, again using the fact that $\varphi_{n} p_{n}=O\left(P_{n}\right)$, we have

$$
\begin{aligned}
\sum_{n=1}^{m} \varphi_{n}^{\delta k+k-1}\left|M_{n, 3}\right|^{k} & =O(1) \sum_{n=1}^{m} \varphi_{n}^{\delta k-1}\left|\lambda_{n}\right|\left|s_{n}\right|^{k} \\
& =O(1) \text { as } m \rightarrow \infty,
\end{aligned}
$$

as in $M_{n, 2}$. Thence, the proof of Theorem 2 is completed. 


\section{CONCLUSION}

In this paper, generalized absolute summability of an infinite series is studied. A general theorem dealing with absolute summability is obtained. For the special cases of $\left(X_{n}\right),\left(\varphi_{n}\right),\left(p_{n}\right)$ and $\delta$, some results can be obtained. If we take $\left(X_{n}\right)$ as a positive non-decreasing sequence in Theorem 2 , we get an another theorem dealing with $\varphi-\left|\bar{N}, p_{n} ; \delta\right|_{k}$ summability of an infinite series. If we take $\varphi_{n}=P_{n} / p_{n}$ in Theorem 2, then we get a known theorem on $\left|\bar{N}, p_{n} ; \delta\right|_{k}$ summability of an infinite series [23]. Also, if we take $\left(X_{n}\right)$ as a positive non-decreasing sequence, $\varphi_{n}=P_{n} / p_{n}$ and $\delta=0$ in Theorem 2, then the condition (10) reduces to the condition (9) and also the conditions $\varphi_{n} p_{n}=O\left(P_{n}\right)$ and (11) are automatically satisfied. Thus, Theorem 2 reduces to Theorem 1 . Finally, if we take $\left(X_{n}\right)$ as a positive non-decreasing sequence, $\varphi_{n}=n, \delta=0$ and $p_{n}=1$ for all values of $n$, then we get a known result of $|C, 1|_{k}$ summability [24].

\section{REFERENCES}

[1] Bari N.K. and Stečkin S.B., Best Approximations and Differential Properties of Two Conjugate Functions, Trudy Moskov. Mat. Obšč., 5 (1956) 483-522.

[2] Seyhan H., On the Local Property of $\varphi-\left|\bar{N}, p_{n} ; \delta\right|_{k}$ Summability of Factored Fourier Series, Bull. Inst. Math. Acad. Sinica, 25-4 (1997) 311-316.

[3] Hardy G.H., Divergent Series, Oxford, Oxford University Press, 1949.

[4] Bor H., On Local Property of $\left|\bar{N}, p_{n} ; \delta\right|_{k}$ Summability of Factored Fourier Series, J. Math. Anal. Appl., 179-2 (1993) 646-649.

[5] Bor H., On Two Summability Methods, Math. Proc. Cambridge Philos. Soc., 97-1 (1985) 147-149.

[6] Flett T.M., On an Extension of Absolute Summability and Some Theorems of Littlewood and Paley, Proc. London Math. Soc. (3), 7 (1957) 113-141.

[7] Bor H., A Note on Absolute Summability Factors, Internat. J. Math. Math. Sci., 17-3 (1994) 479-482.

[8] Bor H. and Seyhan H., On Almost Increasing Sequences and Its Applications, Indian J. Pure Appl. Math., 30-10 (1999) 1041-1046.

[9] Bor H. and Özarslan H.S., On Absolute Riesz Summability Factors, J. Math. Anal. Appl., 246-2 (2000) 657-663.

[10] Bor H. and Özarslan H.S., An Application of Quasi Power Increasing Sequences, Int. Math. J., 2-2 (2002) 187-191.

[11] Bor H. and Özarslan H.S., On an Application of Quasi Power Increasing Sequences, Indian J. Pure Appl. Math., 33-5 (2002), 769-774.

[12] Bor H. and Özarslan H.S., A Note on Absolute Summability Factors, Adv. Stud. Contemp. Math. (Kyungshang) 6-1 (2003) 1-11.

[13] Bor H. and Özarslan H.S., A Note on Absolute Weighted Mean Summability Factors, Cent. Eur. J. Math., 4-4 (2006) 594-599.

[14] Karakaş A., A Note on Absolute Summability Method Involving Almost Increasing and $\delta$ quasi-monotone sequences, Int. J. Math. Comput. Sci., 13-1 (2018) 73-81.

[15] Kartal B., On Generalized Absolute Riesz Summability Method, Commun. Math. Appl., 8-3 (2017) 359-364. 
[16] Özarslan H.S., A Note on $\left|\bar{N}, p_{n} ; \delta\right|_{k}$ Summability Factors, Indian J. Pure Appl. Math., 33-3 (2002) 361-366.

[17] Özarslan H.S., A Note on Quasi Power Increasing Sequences, Indian J. Pure Appl. Math., 34-5 (2003) 727-732.

[18] Özarslan H.S., On $\left|\bar{N}, p_{n} ; \delta\right|_{k}$ Summability Factors, Kyungpook Math. J., 43-1 (2003) 107-112.

[19] Seyhan H. and Sönmez A., On $\varphi-\left|\bar{N}, p_{n} ; \delta\right|_{k}$ Summability Factors, Portugal. Math., 54-4 (1997) 393-398.

[20] Seyhan H., A Note on Absolute Summability Factors, Far East J. Math. Sci., 6-1 (1998) 157-162.

[21] Seyhan H., On the Absolute Summability Factors of Type (A,B), Tamkang J. Math., 30-1 (1999) 59-62.

[22] Mazhar S.M., A Note on Absolute Summability Factors, Bull. Inst. Math. Acad. Sinica, 25-3 (1997) 233-242.

[23] Özarslan H.S., On Almost Increasing Sequences and Its Applications, Int. J. Math. Math. Sci. 255 (2001) 293-298.

[24] Mishra K.N. and Srivastava R.S.L., On Absolute Cesàro Summability Factors of Infinite Series, Portugal. Math., 42-1 (1983-84) 53-61. 